\title{
Conduction velocity and spike configuration in myelinated fibres: computed dependence on internode distance
}

\author{
M. H. BRILL, S. G. WAXMAN, ${ }^{1}$ J. W. MOORE, AND R. W. JOYNER \\ From the Research Laboratory of Electronics, Massachusetts Institute of Technology, Cambridge, Mass., \\ Department of Neurology, Harvard Medical School, Boston, Mass., and Department of Physiology and \\ Pharmacology, Duke University Medical Center, Durham, NC, USA
}

SUMMARY It has been argued theoretically and confirmed experimentally that conduction velocity $(\theta)$ should be proportional to nerve fibre diameter for myelinated fibre tracts, such as normal peripheral nerve, exhibiting 'structural similarity'. In some axons, however, the nodes of Ranvier are more closely spaced than in normal peripheral nerve. Analytic arguments have suggested that when internodal distance $(L)$ alone is changed, the plot of $\theta$ versus $L$ should have a relatively flat maximum. This was confirmed by several previous computer simulations of myelinated axons, but internode lengths of less than half the normal case were not examined. In order to gain insight into impulse propagation in myelinated and remyelinated fibres with short internodal lengths, the present study examines the conduction velocity and spike configuration for a wide range of internodal lengths. As $L$ becomes large, $\theta$ falls and finally propagation is blocked; as $L$ becomes small, $\theta$ decreases more and more steeply. From this, it is predicted that for fibres with very short internodal lengths, small local changes in $L$. should affect substantially the conduction velocity.

Theoretical arguments suggest that neuronal conduction velocity should be proportional to fibre diameter for myelinated fibre tracts which exhibit 'structural similarity' (Rushton, 1951; Goldman and Albus, 1968). One of the conditions of structural similarity is that internode distance should vary linearly with fibre diameter. Normal peripheral nerves satisfy this constraint for a 'similarity class', and the experimental data indicate that conduction velocity is, in fact, approximately proportional to fibre diameter (Hursh, 1939; Gasser and Grundfest, 1939; Tasaki et al., 1943). However, the nodes of remyelinated peripheral fibres are more closely spaced than in normal peripheral nerve (Sanders and Whitteridge, 1946; Schröder, 1975), and they fall into a different similarity class. Their conduction velocities differ from those of normal peripheral axons with the same diameter. Other axons with more closely spaced nodes include remyelinated axons in the central nervous system (Suzuki et al., 1969; Gledhill et al., 1973) as well as preterminal fibres in the neuropil

\footnotetext{
${ }^{1}$ Address for reprint requests: Dr S. G. Waxman, Harvard Neurclogical Unit, Beth Israel Hospital, 330 Brookline Avenue, Boston Mass. 02215, USA.

Accepted 8 February 1977
}

(Waxman, 1970, 1972; Lindsey, 1975), and in some white matter tracts (Meszler et al., 1974).

Huxley and Stämpfli (1949) suggested that conduction velocity in myelinated nerve fibres should have a maximum at a particular internode distance, and that the maximum should be relatively flat. They also predicted that the internodal distances of normal peripheral nerve fibres should fall close to the value for maximum conduction velocity. Several studies (Goldman and Albus, 1968; Hardy, 1971) have tended to confirm this prediction but failed to cover the other similarity classes noted above, because internode lengths used were not short enough (less than one-half normal). Therefore, we have used computer simulations of conduction in myelinated fibres to examine the dependence of conduction velocity and spike configuration on internode distance. Throughout these simulations, the diameter $(d)$, nodal length $(N L)$ and nodal area are fixed and only the internode length $(L)$ is varied (see Table).

\section{Methods}

We used a modification of the model of FitzHugh (1962). The equation to be integrated is 


$$
c(x) \frac{\partial}{\partial t} V(x, t)=\frac{1}{r_{a}} \frac{\partial^{2}}{\partial x^{2}} V(x, t)-i_{m}(x, t)
$$

where:

$V \quad$ is the potential across either the nodal membrane or myelin.

$r_{a}$ is the axial resistance of the fibre per unit length.

$c(x)$ is the capacitance per unit length, the transmyelin capacitance $c_{M}$ in the internodal region, and the nodal capacitance $c_{N}$ at a node of Ranvier.

$i_{m}$ is the ionic current per unit length of membrane. In the internodal regions,

$$
i_{m}(x, t)=g_{M} V(x, t)
$$

where $g_{M}$ is the myelin conductance per unit length. At each node $j$,

$$
i_{m}(x, t)=I_{H H} \cdot \pi d \cdot \frac{N L}{\Delta x}+\frac{\Delta x-N L}{\Delta x}\left[V_{j} \cdot g_{M}\right]
$$

where the current density $I_{H}$ is given by

$$
\begin{array}{r}
I_{H H}=\bar{g}_{N a m_{j}{ }^{3} h_{j}\left[V_{j}-V_{N a}\right]+\bar{g}_{K} n_{j}{ }_{j}\left[V_{j}-V_{K}\right]+} g_{L}\left[V_{j}-V_{L}\right]
\end{array}
$$

where $m_{j}, n_{j}$ and $h_{j}$ satisfy the usual differential equations in time (Hodgkin and Huxley, 1955; FitzHugh, 1962).

These equations were numerically integrated by the Crank-Nicholson method implemented in FORTRAN on a PDP 9 computer. This method has been used for unmyelinated fibres (Moore et al., 1975)

\begin{tabular}{|c|c|c|c|}
\hline Symbol & Explanation & Value & Units \\
\hline $\begin{array}{l}\overline{\mathbf{g}}_{N a} \\
\overline{\mathbf{g}}_{K} \\
\mathbf{g}_{L} \\
\mathbf{V}_{r} \\
\mathbf{V}_{\text {Na }} \\
\mathbf{V}_{K}\end{array}$ & $\begin{array}{l}\text { sodium conductance } \\
\text { potassium conductance } \\
\text { leakage conductance } \\
\text { resting potential } \\
\text { sodium equilibrium potential* } \\
\text { potassium equilibrium } \\
\text { potential }\end{array}$ & $\begin{array}{c}1.2 \\
.09 \\
.02 \\
0 \\
115\end{array}$ & $\begin{array}{l}\mathrm{mho} / \mathrm{cm}^{2} \\
\mathrm{mho} / \mathrm{cm}^{2} \\
\mathrm{mho} / \mathrm{cm}^{2} \\
\mathrm{mV} \\
\mathrm{mV}\end{array}$ \\
\hline $\begin{array}{l}\mathrm{V}_{L} \\
\mathrm{~d}\end{array}$ & leakage equilibrium potential & -.05 & $\mathrm{mV}$ \\
\hline $\begin{array}{l}\text { NL } \\
r_{a}\end{array}$ & $\begin{array}{l}\text { diameter of myelin sheath) } \\
\text { nodal length } \dagger \\
\text { axoplasmic resistance per }\end{array}$ & $\begin{array}{l}10 \\
3.183\end{array}$ & $\underset{\mu \mathrm{m}}{\mu \mathrm{m}}$ \\
\hline g.M & $\begin{array}{l}\text { unit axon length } \ddagger \\
\text { myelin conductance per }\end{array}$ & $1.26 \times 10^{8}$ & $\mathrm{ohm} / \mathrm{cm}$ \\
\hline$c_{M I}$ & myelin capacitance per unit & $5.60 \times 10^{-9}$ & $\mathrm{mho} / \mathrm{cm}$ \\
\hline$c_{N}$ & $\begin{array}{l}\text { axon length } \\
\text { nodal capacitance per unit }\end{array}$ & $1.87 \times 10^{-11}$ & $\mathbf{F} / \mathrm{cm}$ \\
\hline L & internodal distance & $\begin{array}{l}3.14 \times 10^{-\bullet} \\
\text { variable }\end{array}$ & $\mathrm{F} / \mathrm{cm}$ \\
\hline
\end{tabular}
and was adapted for the myelinated fibre by R. W. Joyner This modified Crank-Nicholson method was found to give fast and accurate computation of

\section{Table Parameters}

* All voltage signs are reversed from those of the original HodgkinHuxley formulation.

+ Calculated from nodal area of $100 \mu \mathrm{m}^{2}$.

‡ Calculated from specific axoplasmic resistance of $100 \mathrm{ohm}-\mathrm{cm}$.

$\S$ Calculated from capacitance per unit area of $10^{-6} \mathrm{~F} / \mathrm{cm}^{2}$. impulse propagation and is described in detail by Moore et al. (in preparation). Extensive investigations into the variety of mathematical models for myelinated fibres showed that the impulse propagation velocity was sensitive to the relative values of nodal to internodal characteristics but rather insensitive to changes in the description of the nodal membrane ionic currents.

Because of the insensitivity of propagation velocity to nodal ionic descriptions, we chose to describe the nodal membrane by the most convenient expression for excitable membranes, the Hodgkin-Huxley equations. The parameters used to describe our standard myelinated fibre are given in the Table. The rumbers of sodium and potassium channels were increased by factors of 10 and 2.5 , respectively, to match the nodal conductances measured by voltage clamp methods (Dodge and Frankenhaeuser, 1959). The nodal resting resistance was made compatible with the 55 megohms measured by Tasaki (1955) by increasing $g_{L}$ from 0.003 to $0.02 \mathrm{mho} / \mathrm{cm}^{2}$. Then, to restore the resting potential to $0 \mathrm{mV}$, we changed $V_{L}$ from $+10.6 \mathrm{mV}$ to $-0.05 \mathrm{mV}$. We adjusted the rate constants to $20^{\circ} \mathrm{C}$ by multiplying all rate constants by $3^{(20-6.3) / 10}$. We used a value of $5.60 \times 10^{-9} \mathrm{mho} / \mathrm{cm}$ for $g_{M}$, the myelin conductance, and a value of $1.87 \times 10^{-11} \mathrm{~F} / \mathrm{cm}$ for $c_{M}$, the myelin capacitance. ${ }^{*}$

Our method requires that the space increment $\Delta x$ be larger than the node length $N L$, and we assign a lumped membrane capacitance to space increments containing a node as:

$$
c^{*}=\frac{c_{N} N L+C_{M}(\Delta X-N L)}{\Delta X}
$$

We used ten space segments per internodal distance $L$ for all cases except $L=25 \mu \mathrm{m}$, where it was necessary to reduce the number of segments to five, so $\Delta X$ could exceed $N L$.

Although the method of numerical integration used was found to be unusually stable (compared to other methods which we tried), the largest value of the time increment which could be used without oscillations varied with the internodal length $L$. However, we found that the computations were convergent and stable when $\Delta t$ of $10 \mu \mathrm{s}$ was used for $L \geqslant 1000 \mu \mathrm{m}$, and a $\Delta t$ of $5 \mu \mathrm{s}$ for $L<1000 \mu \mathrm{m}$.

We found that as $L$ was decreased, the current stimulus pulse and the termination artefact spread inward into more and more nodes. For an internodal length greater than $1000 \mu \mathrm{m}$, a steady velocity of propagation obtained within two nodes of either end, and for these computations we used only 12 nodes. As $L$ was decreased from this value, the number of

* These values are $20 \%$ higher than the values used by Moore et al. (in preparation) because of a logarithmic expression for the cylindrical geometry. 
nodes was increased in each case (up to 50 for an $L$ of $25 \mu \mathrm{m})$ so as to obtain a sufficient region of uniform velocity of propagation. Nevertheless, because of the efficiency and stability of our integration method, we were able to reduce the overall computation time several-fold compared to previous methods.

Using these conditions and the above description, we investigated the internodal conduction time and conduction velocity when only the internodal length was varied. The internodal conduction time for a constant velocity impulse was taken as the difference in times at which the rising phase crossed $50 \mathrm{mV}$ at two consecutive nodes. Impulse velocity was inferred from internodal conduction time. The time course of the action potential was plotted for several values of internode length $L$, and the dependence of the action potential shape on this parameter was thus seen.

\section{Results}

Figure 1A shows that the internodal conduction time (ICT) is a monotonically increasing function of internodal distance $L$. For small $L$, the relationship is linear, but it departs from linearity as it goes above $2000 \mu \mathrm{m}$. Figure 1B presents the same data in the form of impulse conduction velocity as a function of $L$. There is a broad maximum between 1000 and $2000 \mu \mathrm{m}$, which corresponds to observations on frog sciatic nerve (FitzHugh, 1962) and agrees with the predictions of Huxley and Stämpfli (1949). Velocity decreases steadily for $L$ above $2000 \mu \mathrm{m}$, and there is conduction failure before $L$ reaches $10000 \mu \mathrm{m}$. Although internodal distances this large have not been observed, the simulation behaviour is interesting to note. For $L=10000 \mu \mathrm{m}$, a spike may be made to spread over the first three or four nodes with very little attenuation but proceeds no further. Apparently a strong step of stimulating current will spread enough so that the currents at the first few nodes exceed the threshold for activity. However, the normal currents generated by nodal action potentials are much smaller and are not able by themselves to sustain sufficient depolarisation and conduction at more distant nodes. For internodal lengths less than $1000 \mu \mathrm{m}$, the conduction velocity decreases dramatically. It is clear from Figure 1B that, for short internode lengths, the velocity is very sensitive to $L$.

Figure $1 \mathrm{C}$ presents the same data as the travel time per unit distance $(1 / \theta)$ as a function of the logarithm of $L$ (to show the effect of relative changes of $L$ ). For small values of $L$, the travel time depends almost linearly on equal relative changes in $L$. Of course, the travel time is least sensitive to $L$ in the $1000-2000 \mu \mathrm{m}$ region.

Having carried out these computations for only one value of $d\left(10 \mu \mathrm{m}\right.$, which we henceforth call $\left.d_{0}\right)$,

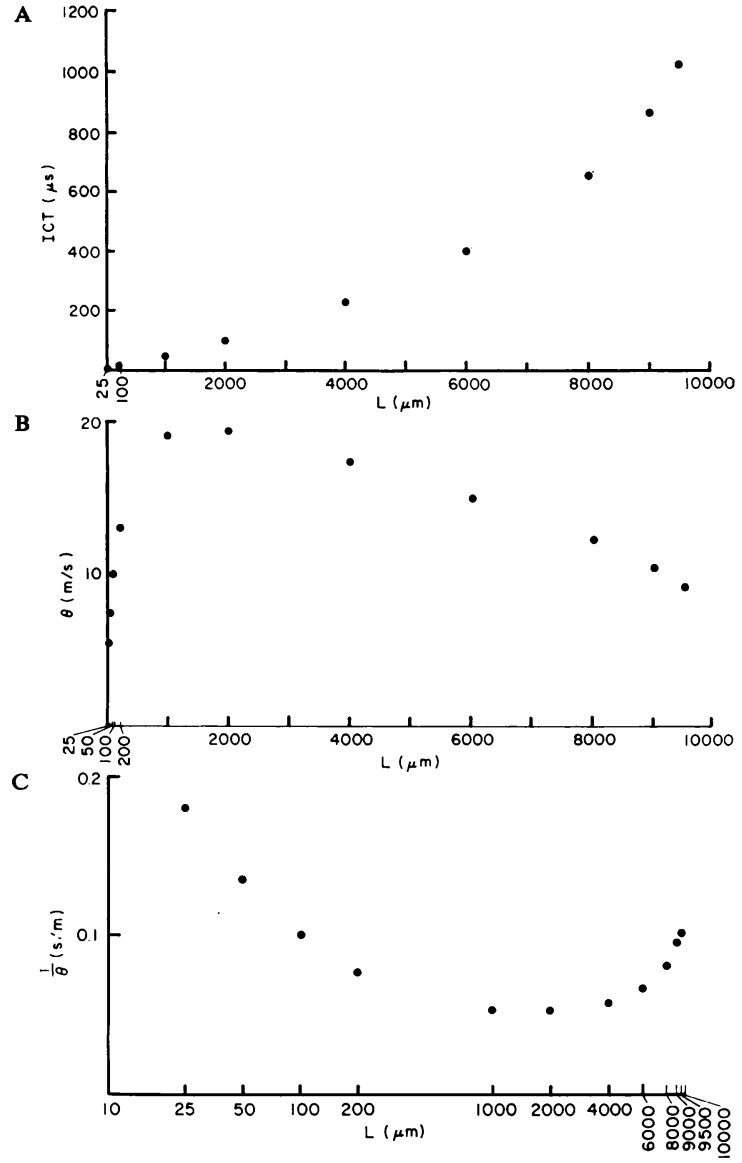

Fig. 1 (A) Plot of the internodal conduction time (ICT) of a steadily propagating action potential versus internodal distance $L$. (B) Plot of the velocity $\theta$ of a steadily propagating action potential versus internodal distance $L$. (C) Plot of $1 / \theta$ for a steadily propagating action potential versus $L .1 / \theta$ represents the time for an impulse to travel one metre, and $L$ is displayed logarithmically to show the change in $1 / \theta$ accompanying relative changes in $L$.

we can take any point on the curve to represent a different 'similarity class'. By using Rushton's (1951) correspondence principle, we can interpret Figs. 1B and 1C more generally for different axon diameters. Rushton postulated that peripheral nerve fibres fall into an equivalence class in which fibres exhibit 'dimensional similarity'. Dimensional similarity requires that internode length, myelin thickness, and nodal area vary directly with fibre diameter. Given a class of fibres that exhibit dimensional similarity and in which the intrinsic membrane properties are all the same, Rushton showed that internodal conduction time should be the same for all fibres of the class- 
that is, conduction velocity varies linearly with fibre diameter.

Therefore, given a fibre with certain internode length $L$ and impulse velocity $\theta$, we can generalise Figs. $1 \mathrm{~B}$ and $1 \mathrm{C}$ to fibres of other diameters by scaling $\theta$ and $L$ by $d / d_{o}$. It should be noted that dimensional similarity breaks down when nodal length is not negligible compared to internodal length, so it may not apply to fibres with the lowest values of the ratio L/d (Waxman and Bennett, 1972). Furthermore, Coppin and Jack (1972) have shown that the internodal conduction times of small peripheral myelin-
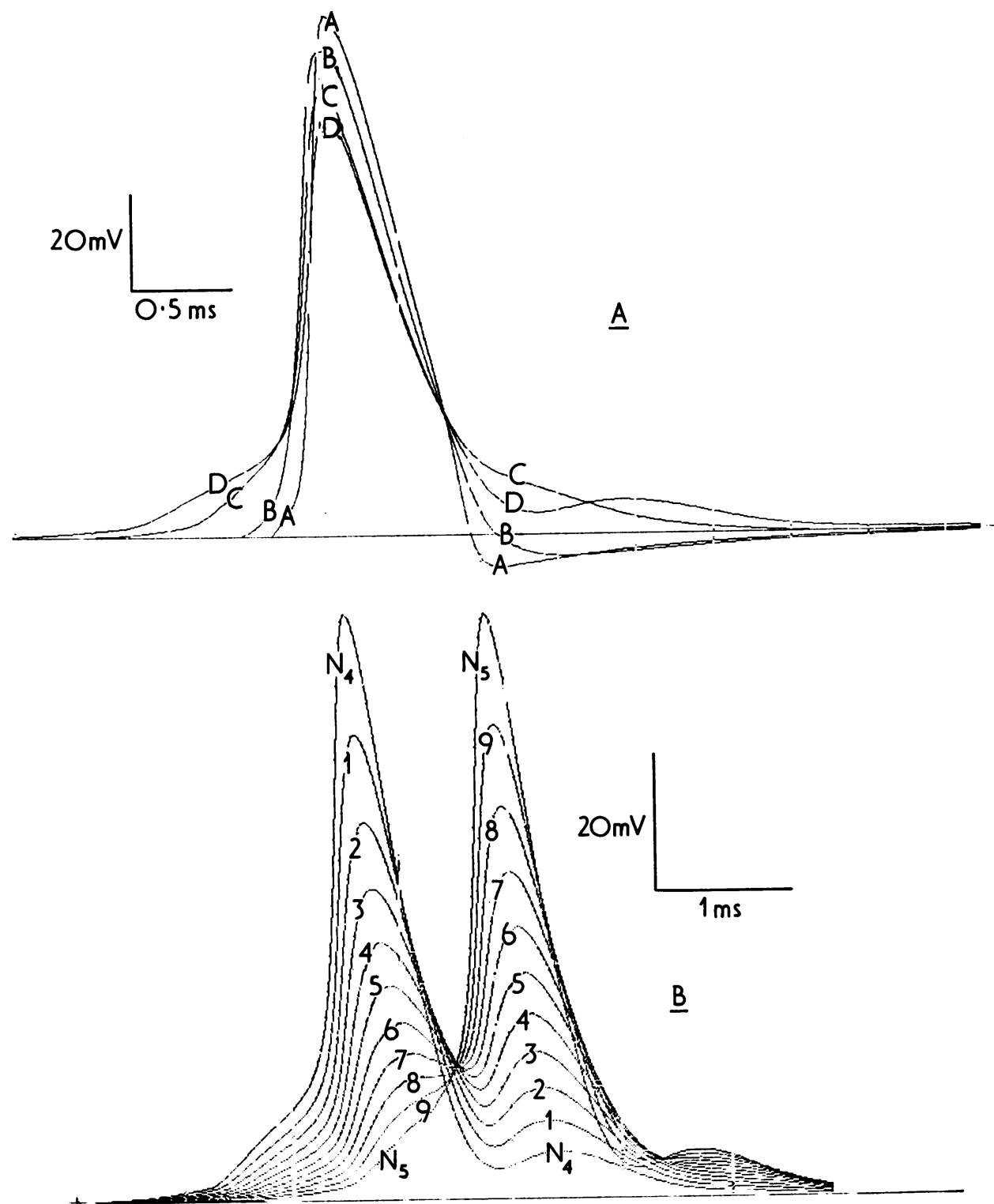

Fig. 2 (A) Plot of voltage versus time for steadily propagating action potentials corresponding to several values of $L$. For ease of comparison, peak times are made to coincide. Curves $A-D$ represent $L=50,2000$, 8000 , and $9500 \mu \mathrm{m}$, respectively; $(B)$ the potential at nine internodal points (labelled 1-9) between nodes 4 and $5\left(N_{4}, N_{5}\right)$ for the case $L=9500 \mu m$ (see text). 
ated fibres deviate from the constant value predicted from dimensional similarity. Nevertheless, predictions derived from dimensional similarity do appear to apply to large myelinated fibres (FitzHugh, 1973; Waxman, 1975).

Figure 2A presents the time course of an action potential propagating at constant velocity, for several values of $L$. The shape and amplitude of the impulse depends strongly on $L$. For $L=50 \mu \mathrm{m}$ (A), the falling of the action potential shows a slight 'gratuitous hump' which is characteristic of action potentials calculated from the Hodgkin-Huxley (1952) equations. As the internodal length is increased, the hump disappears by $2000 \mu \mathrm{m}$ (B), and by $8000 \mu \mathrm{m}$ (C) the undershoot is masked by a positive shoulder late in the falling phase of the action potential. For lengths just short of maximum $(9500 \mu \mathrm{m}, \mathrm{D})$, this shoulder has become a distinct hump. To ascertain the origin of this shoulder, we computed the potential at each of nine equidistant internodal points between nodes 4 and 5. As shown in Fig. 2B, the secondary hump is the result of electrotonus from the spike at node 5 .

\section{Discussion}

From the maximum in Fig. 1B and the minimum in Fig. $1 C$, it is clear that fibres with $L / d=150$ do not suffer large changes in $\theta$ when $L / d$ is changed modestly. This is consistent with the observation that, in remyelinated peripheral axons as compared to control axons, conduction velocity is reduced, but to a statistically insignificant degree (Sanders and Whitteridge, 1946). On the other hand, the simulations predict that fibres with small $L / d$ would be quite sensitive to variations in $L / d$. This sensitivity might provide insight as to a possible physiological significance of the fact that some normal (Waxman, 1970; Meszler et al., 1974; Lindsey, 1975) and remyelinated (Gledhill et al., 1973) CNS fibres have an $L / d$ ratio that is much less than for normal peripheral nerve fibres. Because the nerve impulse velocity is insensitive to small changes in $L / d$, there would not seem to be any signalprocessing significance to minor local changes in $L$ in peripheral fibres. On the other hand, for CNS fibres with small $L / d$ ratios, we cannot ignore the effects of small local changes in $L$ on signal processing, because the velocity of propagation depends so dramatically on $L$. In those central fibres where $L / d$ is normally very small (Waxman, 1970, 1972; Meszler et al., 1974; Lindsey, 1975), local changes in $L$ may allow fine tuning of the times of arrival of impulses at synapses or provide a convenient way of presetting routedependent travel times in the central nervous system (Bennett, 1968; Waxman, 1975). For central remyelinated fibres where $L / d$ is substantially reduced (Gledhill et al., 1973), the present results would also suggest a marked reduction in conduction velocity.

This work was supported in part by grants from the National Institutes of Health (NS-12307, TO1EY00090, NS-03437, NS-11613), the Health Sciences Fund (78-10), and by a Research Career Development Award (KO4-NS-00010) to SGW. We thank Professor J. Y. Lettvin for helpful comments and encouragement, and Professor M. Eden for generously making computer time available.

\section{References}

Bennett, M. V. L. (1968). Neural control of electric organs. In The Central Nervous System and Fish Behavior, pp. $147-169$. Edited by D. Ingle. The University of Chicago: Illinois.

Coppin, C. M. L., and Jack, J. J. B. (1972). Internodal length and conduction velocity of cat muscle afferent nerve fibres. Journal of Physiology, 222, 91-93p.

Dodge, F. A., and Frankenhaeuser, B. (1959). Sodium currents in the myelinated nerve fibre of Xenopus laevis investigated with the voltage clamp technique. Journal of Physiology, 148, 188-200.

FitzHugh, R. (1973). Dimensional analysis of nerve models. Journal of Theoretical Biology, 40, 517-541.

FitzHugh, R. (1962). Computation of impulse initiation and saltatory conduction in a myelinated nerve fiber. Biophysical Journal, 2, 11-21.

Gasser, H. S., and Grundfest, H. (1939). Axon diameters in relation to the spike dimensions and the conduction velocity in mammalian A fibres. American Journal of Physiology, 127, 393-414.

Gledhill, R. F., Harrison, B. M., and McDonald, W. I. (1973). Pattern of remyelination in the CNS. Nature, 244, 443-444.

Goldman, L., and Albus, J. S. (1968). Computation of impulse conduction in myelinated fibers; theoretical basis of the velocity-diameter relation. Biophysical Journal, 8, 596-607.

Hardy, W. L. (1971). Computed dependence of conduction speed in myelinated axons on geometric parameters. Biophysical Society Abstracts, 11, 238a.

Hodgkin, A. L., and Huxley, A. F. (1952). A quantitative description of membrane current and its application to conduction and excitation in nerve. Journal of Physiology, 117, 500-544.

Hursh, J. B. (1939). Conduction velocity and diameter of nerve fibers. American Journal of Physiology, 127, 131-139.

Huxley, A. F., and Stämpfli, R. (1949). Evidence for saltatory conduction in peripheral myelinated nerve fibres. Journal of Physiology, 108, 315-339.

Lindsey, B. G. (1975). Fine structure and distribution of axon terminals from the cochlear nucleus on neurons in the medial superior olivary nucleus of the cat. Journal of Comparative Neurology, 160, 81-104.

Meszler, R. M., Pappas, G. D., and Bennett, M. V. L. (1974). Morphology of the electromotor system in the spinal cord of the electric eel, Electrophorus electricus. Journal of Neurocytology, 3, 251-261. 
Moore, J. W., Ramón, F., and Joyner, R. W. (1975). Axon voltage-clamp simulations I. Methods and tests. Biophysical Journal, 15, 11-24.

Rushton, W. A. H. (1951). A theory of the effects of fibre size in medullated nerve. Journal of Physiology, 115, 101-122.

Sanders, F. K., and Whitteridge, D. (1946). Conduction velocity and myelin thickness in regenerating nerve fibres. Journal of Physiology, 105, 152-174.

Schröder, J. M. (1975). Degeneration and regeneration of myelinated nerve fibers in experimental neuropathies. In Peripheral Neuropathy, pp. 337-362. Edited by P. J. Dyck, P. K. Thomas, and E. M. Lambert. Saunders: Philadelphia.

Suzuki, K., Andrews, J. M., Waltz, J. M., and Terry, R. D. (1969). Ultrastructural studies of multiple sclerosis. Laboratory Investigation, 20, 444-454.

Tasaki, I. (1955). New measurements of the capacity and the resistance of the myelin sheath and the nodal membrane of the isolated frog nerve fiber. American Journal of Physiology, 181, 639-650.

Tasaki, I., Ishii, K., and Ito, H. (1943). On the relation between the conduction-rate, the fibre-diameter and the internodal distance of the medullated nerve fibre. Japanese Journal of Medical Science III, Biophysics, 9, 189-200.

Waxman, S. G. (1975). Integrative properties and design principles of axons. International Review of Neurobiology, 18, 1-40.

Waxman, S. G. (1972). Regional differentiation of the axon: A review with special reference to the concept of the multiplex neuron. Brain Research, 47, 269-288.

Waxman, S. G. (1970). Closely spaced nodes of Ranvier in the teleost brain. Nature, 227, 283-284.

Waxman, S. G., and Bennett, M. V. L. (1972). Relative conduction velocities of small myelinated and nonmyelinated fibres in the central nervous system. Nature New Biology, 238, 217-219. 